\title{
44496 - CREATING A CULTURE OF RESEARCH IN ANESTHESIOLOGY: A SURVEY
}

\author{
Michael Murphy, Dalhousie University, Halifax, NS, Canada; \\ Orlando Hung, Dalhousie University; \\ Debbie Tamlyn, Dalhousie University;
}

\section{INTRODUCTION:}

Leadership, vision, collaboration and resources are needed to enhance research in Anesthesia. Alternative Funding Programs (AFP) are critical in providing time and resources to foster a research agenda The objective of this project was to obtain input from departmental team members to serve as the basis of creating an enhanced focus on research and evidence based practice consistent with requirements of a new AFP Agreement.

\section{METHODS:}

An interview guide consisting of 14 questions was developed to gain information on topics such as: research strengths and weaknesses; incentives and barriers to research; fostering research in residency programs; and how best to promote research collaboration. Face-to-face interviews were conducted with 25 departmental representatives including specialists, research support staff and trainees. The interview findings were discussed in a one-day departmental retreat.

\section{RESULTS:}

The following main themes emerged from the interviews and retreat: the importance of having a clear research plan and dedicated space and resources (infrastructure); the plan must build upon current research strengths (e.g. in Airway Management, Pharmacology, Pain Management and Medical Informatics); the importance of having equal compensation for research and clinical practice; methods for outcome measures and evaluation of productivity are needed; research fellowships and awards are important in promoting research for residents; coordination and collaboration among support staff needs to be enhanced; technology support and the use of webbased communication for management and research communication and databases are essential; expectations for resident education and research must be clearly articulated; the significance and expectations for appointments and promotion must be clearly defined.

\section{DISCUSSION:}

This report presents interview findings and provides an overview of the Strategic Research Plan that is being developed and implemented. There has been increased pressure in the last few years to address wait times for surgery. This has meant less time for research and a diminished emphasis on the research agenda. To reverse this trend, it is essential to take into account the perspectives of the entire anesthesia team and to develop a strategic research plan with timelines and resources. Leadership, vision and team involvement are necessary to create a new culture of research. 